\title{
Global Theory to Understand Toroidal Drift Waves in Steep Gradient
}

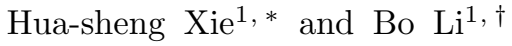 \\ ${ }^{1}$ Fusion Simulation Center, State Key Laboratory of Nuclear Physics and Technology, \\ School of Physics, Peking University, Beijing 100871, China
}

(Dated: July 15, 2021)

\begin{abstract}
Toroidal drift waves with unconventional mode structures and non-ground eigenstates, which differ from typical ballooning structure mode, are found to be important recently by large scale global gyrokinetic simulations and especially become dominant at strong gradient edge plasmas [cf., Xie and Xiao, Phys. Plasmas, 22, 090703 (2015)]. The global stability and mode structures of drift wave in this steep edge density and temperature gradients are examined by both direct numerical solutions of a model two-dimensional eigen equation and analytical theory employing WKB-ballooning approach. Theory agrees with numerical solutions quite well. Our results indicate that (i) non-ground eigenstates and unconventional mode structures generally exist and can be roughly described by two parameters 'quantum number' $l$ and ballooning angle $\vartheta_{k}$, (ii) local model can overestimate the growth rate largely, say, $>50 \%$, and (iii) the narrow steep equilibrium profile leads to twisting (triangle-like) radial mode structures. With velocity space integral, semi-local theory predicts that the critical jump gradient of the most unstable ion temperature gradient mode from ground state $l=0$ to non-ground state $l=1$ is $L_{T}^{-1} R \sim 50$. These features can have important consequences to turbulent transport.
\end{abstract}

PACS numbers: 52.35.Py, 52.30.Gz, 52.35.Kt

\section{INTRODUCTION AND MOTIVATION}

Drift waves 1 ] widely exist in nonuniform magnetized plasmas and are thought to be the dominant turbulent transport mechanism for particle, energy and momentum. Although it has been known theoretically 2 4 for decades that there exists many eigenstates for toroidal drift waves, such as ion temperature gradient mode (ITG) and trapped electron mode (TEM), most efforts focused on the ground state branch ('quantum number' $l=0$, aka., fundamental branch) in the past due to that it is usually the most significant branch in the experiments. Recently, under strong gradient edge plasma parameters, it was found by global gyrokinetic simulations that the most unstable branch can jump from ground state to non-ground states 5 , 41, i.e., the most unstable branch is non-ground states and with also usually unconventional mode structures, whereas the conventional mode structure has typical ballooning structure which localizes at the outboard of poloidal plane. The modes jump and unconventional mode structures have also been reported in local gyrokinetic simulations 6, 7]. The modes jump here is eigenstates jump (e.g, from one TEM to another TEM) in contrast to the jump from one kind of mode to another, such as from ITG to TEM 8 and to kinetic ballooning mode (KBM) 9 . Global unconventional mode structures are also reported in Refs. [1013, but are not identified as eigenstates jump. In Refs. 57. 41, the new physics happens at steep gradient edge region $\left(R L_{T}^{-1}>40\right.$, where $R$ is major radius and $L_{T}^{-1}$ is

*Email: huashengxie@gmail.com

†Corresponding author. Email: bli@pku.edu.cn temperature gradient scale length); whereas most previous works study core plasma with $R L_{T}^{-1}<20$, which is the reason why the unconventional mode structures and eigenstates jump are only reported very recently.

Considering that most previous works [1, 3, 4, 8, 14 18. of drift waves focus on the fundamental solution with weak gradient, the non-ground solutions at strong gradient required further theoretical studies to provide a complete picture to understand the most general mode structure and the distributions and transitions of eigenstates (eigenvalue solutions). The framework to understand those recently and future gyrokinetic simulation and experimental results should include: global solutions instead of only local solutions, eigenstates jump, critical jump gradient (hereafter, we discuss the critical gradient for the most unstable mode jump from ground eigenstate to non-ground states, not the usual critical gradient for the mode from stable to unstable), unconventional mode structures, possible electromagnetic (EM) effects [11, consequences (e.g., to turbulent transport) and physical understanding. These should be resolved one-by-one.

The reasons why local solutions are not adequate are mainly due to two reasons. The first is that the mode structures from local solution are not intuitive and may not be able to used to compared with global simulations or experiments directly, which will also affect the nonlinear consequences such as turbulent transport. For example, at least a transformation (in generally, not straightforward) to include the second dimension solution from the 1D (one dimensional) local to 2D (two dimensional) global mode structure are required, cf. Refs. [17, 19]. The second reason is more important to motivate the present work: at strong gradient the local solutions may not be quantitatively correct and thus cannot be used to quan- 
titatively compare with experiments. This can be seen in a benchmark effort in Ref.77. We can see in Fig.14 of Ref.[7] that at weak gradient different local gyrokinetic codes can have good agreements at real frequency and growth rate but at strong gradient pedestal parameters the agreement breaking down. This deviation in local codes can come from either different models (e.g., model equation or equilibrium implementation) or the breaking down of the local assumption. Further study is required to identify the validation of the local model for study the strong gradient edge parameters.

In this work, we solve a global $2 \mathrm{D}$ toroidal drift wave model equation both analytically and numerically, as one step to understand the complete picture of drift wave in steep gradient. The results can understand several aspects of the simulations in Ref. [5] and is an extension of the model theory in that work, especially, which shows that non-ground eigenstates and unconventional mode structures generally exist. Another interesting feature in Ref. [5] (although not be emphasized there) is twisting (triangle-like) radial mode structure. Recently, the twisting mode have also been found in experiments and simulations for energetic particle (EP) excited reversed shear Alfvén eigenmode (RSAE) [20] and beta-induced Alfvén eigenmode (BAE) 21 23]. Global theory 24] explains that the twisting mode is due to anti-Hermitian contributions from wave-energetic particle resonance. Without $\mathrm{EP}$, new theory is required to understand the twisting radial mode structures in Ref. [5]. This is another motivation of this work. We have also noticed that global 2D numerical solutions of model drift wave equation are also reported in Ref.[12, 13] for fundamental solution, where modes localized at $\theta \simeq \pm \pi / 2$ are found. Later we will conclude that those solutions are merely one of series solutions and are not the unconventional solutions in steep gradient as reported in global simulations [5, 10. Rotation and shear flow $13,17,25]$ can also modify the typical ballooning structure but will be neglected in the present study.

In the following sections, Sec.II gives the model equation and summarizes the ballooning representation theory. Sec III gives the local and global analytic solutions. Sec IV focuses on the global numerical solutions. Sec V studies the global gradient profile effects. SecVI uses a semi-local kinetic model with velocity space integral to give a more accurate eigenstates jump critical gradient. Sec VII summarizes the present study.

\section{MODEL EQUATION AND BALLOONING REPRESENTATION}

To focus on the qualitative behavior of the general mode structures and eigenstates, we start from a simple $i \delta$ drift wave model, which can be used to model ion temperature gradient mode (ITG) and trapped electron mode (TEM), and has been widely used for theoretical studies (cf. [5, 13, 18]). For a large aspect ratio, circular cross section symmetric tokamak equilibrium, the starting 2D equation (after Fourier decomposition of toroidal direction and time dependence $\sim e^{i n \zeta-i \omega t}$ ) for electrostatic fluctuations potential $\delta \phi(r, \theta)$ is [18]

$$
\begin{gathered}
\rho_{s}^{2} \frac{\partial^{2} \delta \phi}{\partial x^{2}}-b_{s} \delta \phi-\left(\frac{\omega_{* e}}{\omega} \frac{\epsilon_{n}}{q k_{\theta} \rho_{s}}\right)^{2}\left(\frac{\partial}{\partial \theta}+i k_{\theta} s x\right)^{2} \delta \phi+ \\
{\left[\frac{\omega_{* e}-\omega\left(1-i \delta_{e}\right)}{\omega_{* e} \eta_{s}+\omega}\right] \delta \phi-2 \frac{\omega_{* e}}{\omega} \chi \epsilon_{n}\left(\cos \theta+\sin \theta \frac{i}{k_{\theta}} \frac{\partial}{\partial x}\right) \delta \phi=0,}
\end{gathered}
$$

where $\rho_{s} \equiv \sqrt{m_{i} T_{e}} / e B, T_{e}$ is electron temperature, $m_{i}$ is ion mass, $e$ is the unit charge, $B$ is the magnetic field, $k_{\theta} \equiv n q / r$ is the poloidal wave number, $n$ is toroidal mode number, $b_{s} \equiv k_{\theta}^{2} \rho_{s}^{2}, \omega_{s} \equiv c_{s} / R, c_{s} \equiv \sqrt{T_{e} / m_{i}}$, $\tau \equiv T_{e} / T_{i}, q \equiv r B_{\zeta} / R B_{\theta}$ is safety factor, $R$ is major radius, $s \equiv(r / q)(d q / d r)$ is shear, $\omega_{* e} \equiv k_{\theta} T_{e} /\left(e B L_{n}\right)$, $L_{n}^{-1} \equiv-\partial \ln n_{0} / d r$ is density gradient length scale, $\epsilon_{n} \equiv L_{n} / R, \eta_{s} \equiv\left(1+\eta_{i}\right) / \tau, \eta_{i} \equiv L_{T_{i}}^{-1} / L_{n}^{-1}$, and $\delta_{e}$ is nonadiabtic electron response. The poloidal angle $\theta=0$ is at the outboard mid-plane of the torus, $x=r-r_{s}$ is the radial distance from the local rational surface $r=r_{s}$ with $n q\left(r_{s}\right)=m_{0}$ (integer). The complex mode frequency $\omega \equiv \omega_{r}+i \gamma\left(\gamma \equiv \omega_{i}\right)$. In Ref.[18, temperature gradient length scale $L_{T}^{-1} \equiv-\partial \ln T_{e} / d r=0$, i.e., $\eta_{s}=1 / \tau$. Parameter $\chi$ is an artificial coupling strength, default $\chi=1$; whereas the model reduces to cylinder drift waves at $\chi=0$. To study the equilibrium gradient profile effect, we will let $L_{n}^{-1}=L_{n}^{-1}(x)$, which also leads to $\epsilon_{n}=\epsilon_{n}(x)$ and $\omega_{* e}=\omega_{* e}(x)=\omega_{s} k_{\theta} \rho_{s} \epsilon_{n}^{-1}(x)=\omega_{s 0} \epsilon_{n}^{-1}(x)$, where $\omega_{s 0} \equiv \omega_{s} k_{\theta} \rho_{s}$. For simplicity, all other parameters (say, $\left.q=q_{0}, s, k_{\theta} \rho_{s}, \tau, \eta_{i}, \ldots\right)$ in Eq.(1) are taken independently on radial coordinate $x$ in the following study (except in SecIV). Usually, $\delta_{e}$ should be an integral operator, but we will take it as a non-negative constant here.

Using Fourier $\delta \phi(x, \theta)=\sum_{m} u_{m}(x) e^{-i m \theta}$ to rewrite Eq.(1), the $2 \mathrm{D}$ equation yields

$$
\begin{aligned}
& \left\{k^{2} s^{2} \frac{d^{2}}{d z^{2}}+\frac{1}{k^{2} q^{2} \omega^{2}}(z-m)^{2}-k^{2}-\frac{\omega\left(1-i \delta_{e}\right)-\epsilon_{n}^{-1}}{\omega+\eta_{s} \epsilon_{n}^{-1}}\right\} u_{m} \\
& -\chi \frac{1}{\omega}\left[\left(1+s \frac{d}{d z}\right) u_{m+1}+\left(1-s \frac{d}{d z}\right) u_{m-1}\right]=0
\end{aligned}
$$

with $u_{m} \equiv u_{m}(z)=\delta \phi_{m}, z \equiv k_{\theta} s x=n q^{\prime} x, \delta m=$ $m-m_{0}, k \equiv k_{\theta} \rho_{s}$ and $\omega$ have been normalized by $\omega_{s 0}$. The only differences from the equation in Ref. [5] are the additional parameters $i \delta_{e}, \tau$ and $\chi$.

Considering that usually $u_{m}(x)$ in Eq.(2) is nearly translational invariant under $(m, x) \rightarrow\left(m+1, x+\Delta r_{n}\right)$, where $\Delta r_{n}=1 / n q^{\prime} \ll L_{e q}$ is distance between mode rational surfaces and $L_{e q}$ is slow varying equilibrium length scale, we can assume $u_{m}(x)=u_{0}\left(x-\delta m / n q^{\prime}\right) A(x)$, with $A(x)=\bar{A}(x) e^{i m \vartheta_{k}}$. Here, $\bar{A}(x)$ is slow equilibrium length scale amplitude variation, and $e^{i m \vartheta_{k}}$ can represent the phase variation between neighboring Fourier components. The Fourier form of radial $u_{0}(x)$ can be $u_{0}(x)=\int_{-\infty}^{\infty} e^{i m_{0} \eta} e^{-i n q^{\prime} x \eta} f(\eta) d \eta$, thus finally we obtain the ballooning representation [18, 26, 27] (assumed 


$$
\begin{aligned}
\left.n \gg 1, q \simeq q_{0}+q^{\prime} x\right) \\
\delta \phi(x, \theta)=\sum_{m} e^{-i m\left(\theta-\vartheta_{k}\right)} \int_{-\infty}^{\infty} d \eta e^{i\left(m-n q^{\prime} x\right) \eta} \bar{A}(x) f(\eta) \\
=A(x) \sum_{m} e^{-i m \theta} \int_{-\infty}^{\infty} d \eta e^{i(m-n q) \eta} f(\eta)
\end{aligned}
$$

Comparing the two forms in Eq. 3), we can see that the $\vartheta_{k}$ can represent also: (a) the wave number of radial envelope by WKB approximation $A(x)=e^{i n \int \vartheta_{k}(x) q^{\prime} d x}$ $\left(\delta m \simeq n q^{\prime} x\right)$ and $\vartheta_{k}=k_{r} / n q^{\prime}$, and (b) the poloidal peaking angle of perturbation in $2 \mathrm{D}(r, \theta)$ plane from $e^{-i m\left(\theta-\vartheta_{k}\right)}$.

Keeping only the lowest order transformation $u_{m} \rightarrow$ $f(\eta), \partial / \partial z \rightarrow-i\left(\eta-\vartheta_{k}\right),(m-z) \rightarrow-i \partial / \partial \eta$, we can obtain a local $1 \mathrm{D}$ ballooning space equation from Eq. (2)

$$
\begin{aligned}
& \left\{\frac{1}{q^{2} k^{2}} \frac{d^{2}}{d \eta^{2}}+\left[\omega^{2} \frac{\omega\left(1-i \delta_{e}\right)-\epsilon_{n}^{-1}}{\omega+\eta_{s} \epsilon_{n}^{-1}}+\omega^{2} k^{2}[1+\right.\right. \\
& \left.\left.\left.s^{2}\left(\eta-\vartheta_{k}\right)^{2}\right]+2 \omega K\right]\right\} f\left(\eta, \vartheta_{k}\right)=0,
\end{aligned}
$$

where $K\left(\eta, \vartheta_{k}\right) \equiv \chi\left[\cos \eta+s\left(\eta-\vartheta_{k}\right) \sin \eta\right]$ is from the torodial coupling terms $u_{m \pm 1}, f\left(\eta, \vartheta_{k}\right)$ is the electrostatic potential, $\eta \in(-\infty, \infty)$ is the ballooning poloidal angle coordinate, $f(\eta)$ is Fourier transform of the radial structure $u_{0}(n q-m)$. The coordinate $\eta$ can also be seen as field line coordinate (parallel direction) due to the mapping relation $\nabla_{\|} \delta \phi(r, \theta, \zeta, t) \rightarrow(n q-m) u_{0}(x) \rightarrow \partial_{\eta} f(\eta)$. Here, the ballooning angle parameter $\vartheta_{k} \in[-\pi, \pi]$ [generally, $\vartheta_{k}=\vartheta_{k r}+i \vartheta_{k i}$ is complex, but the imaginary part $i \vartheta_{k i}$ can be absorbed to $\left.\bar{A}(x)\right]$ is to be determined from high order 18] (2D) theory but can be treated as a parameter in 1D model. Eqs. (1)-(4) (or small variation) have been studied by many authors $3-5,12,14,18,28$. In this work, we will use them to study the multi-eigenstates and global unconventional mode structures in steep gradient.

Eq. (4) can be rewritten to polynomial form

$$
\left\{\omega^{3} a_{3}+\omega^{2} a_{2}+\omega a_{1}+a_{0}\right\} f=0,
$$

where $a_{3}=\left(1-i \delta_{e}\right)+k^{2}\left[1+s^{2}\left(\eta-\vartheta_{k}\right)^{2}\right], a_{2}=\eta_{s} k^{2} \epsilon_{n}^{-1}[1+$ $\left.s^{2}\left(\eta-\vartheta_{k}\right)^{2}\right]+2 K-\epsilon_{n}^{-1}, \quad a_{1}=\frac{1}{q^{2} k^{2}} \frac{d^{2}}{d \eta^{2}}+2 \eta_{s} \epsilon_{n}^{-1} K$, $a_{0}=\eta_{s} \frac{\epsilon_{n}^{-1}}{q^{2} k^{2}} \frac{d^{2}}{d \eta^{2}}$. Eq. 2 can also easily be written to polynomial form (Appendix A). These polynomial differential equations can be solved numerically by companion matrix method[5] to obtain all solutions. Fig, 1 shows a typical case of the distributions of the 1D numerical solutions of the Eq. (5) with central difference method and zero boundary condition, where we take $\chi=0.2$ is to make the TEM solution more clearly, otherwise it is usually difficult to be distinguished from the background solutions. The matrix system is $3 N_{\eta} \times 3 N_{\eta}$ dimensions, thus contains $3 N_{\eta}$ solutions, where $N_{\eta}$ is grid numbers of $\eta$. We will know later that $N_{\eta}$ is relevant to quantum number $l_{\eta}$ and 3 means three branches, one is TEM (electron drift wave) and another two are ITGs (but only

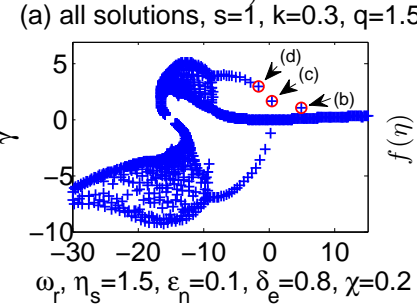

(c) ITG, I=0

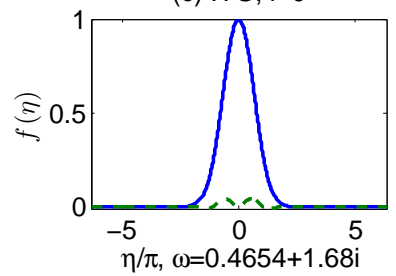

(b) TEM, l=0



(d) ITG, I=1

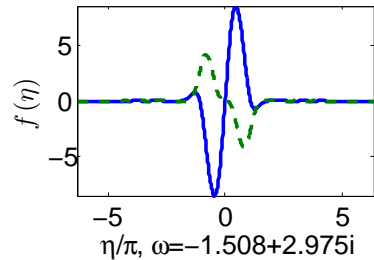

FIG. 1: The distributions of all solutions (a) of Eq. [5], with $s=1.0, k=0.3, q=1.5, \epsilon_{n}=0.1, \eta_{s}=1.5, \delta_{e}=0.8, \chi=0.2$, $\vartheta_{k}=0$ and grid parameters $N_{\eta}=768, \eta \in\left[-\eta_{\max }, \eta_{\max }\right]$, $\eta_{\max }=20$. (b), (c), (d) show the mode structures of TEM and ITG with quantum number $l=0,1$. The frequencies of them are located by red circles at (a).

the unstable branch is our major interest). The positive frequency (electron diamagnetic direction instead of ion diamagnetic direction) of ITG in Fig 1 is due to the modification effects of non-zero $i \delta_{e}$ term. Positive ITG frequency and negative TEM frequency have also been reported in more accurate gyrokinetic models 29, 30. It is also readily to show that the solutions of Eq. (5) have several symmetric properties: (a) For $\delta_{e}=0$, if $(\omega, f)$ is a solution, $\left(\omega^{*}, f^{*}\right)$ is also a solution, where asterisk denotes complex conjugation; (b) For $\vartheta_{k}=0$, the solution will be either odd or even parity. The solutions in Ref. [5] satisfy the above two properties. Another uncertainty is that if $f(\eta)$ is a solution, $c f(\eta)$ is also a solution, where $c$ is arbitrary complex number. To eliminate this uncertainty in mode structure, say for even mode, we can 'normalize' $f \rightarrow c f(\eta) / f(0)$, with e.g., $c=1+0 i$. The normalization of odd modes in this work is slightly arbitrary.

\section{ANALYTICAL LOCAL AND GLOBAL SOLUTIONS}

A physical meaningful solution should satisfy the decaying boundary condition: for the 1D Eq. (5) is $f(\eta \rightarrow$ $\pm \infty) \rightarrow 0$; for the 2D Eq. (2) is $u_{m}(z \rightarrow \pm \infty) \rightarrow 0$. Note also that for the $2 \mathrm{D}$ equation, the boundary condition for $\theta$ (poloidal) direction is periodic. 
TABLE I: Analytical solutions vs. numerical solutions, parameters are same as in Fig 1 .

\begin{tabular}{|c|c|c|c|c|c|c|c|c|}
\hline$\omega$ & TEM $l=0$ & TEM $l=1$ & $\mathrm{ITG}^{+} l=0$ & $\mathrm{ITG}^{+} l=1$ & $\mathrm{ITG}^{+} l=2$ & $\mathrm{ITG}^{-} l=0$ & other $l=0$ & other $l=0$ \\
\hline Eq. A1) & $5.065+1.214 \mathrm{i}$ & $7.647-0.586 \mathrm{i}$ & $0.519+2.373 \mathrm{i}$ & $-1.143+3.413 \mathrm{i}$ & $-2.183+3.757 \mathrm{i}$ & $0.601-1.694 \mathrm{i}$ & $4.424+5.348 \mathrm{i}$ & $-0.770-0.020 \mathrm{i}$ \\
\hline Eq.55 w/ apx & $5.065+1.214 \mathrm{i}$ & - & $0.519+2.373 \mathrm{i}$ & $-1.142+3.413 \mathrm{i}$ & $-2.182+3.757 i$ & $0.601-1.694 \mathrm{i}$ & - & - \\
\hline
\end{tabular}

\section{A. Analytical limit of local solution}

At analytical limit, assuming that the mode is located around $\eta \simeq \vartheta_{k},\left|\eta-\vartheta_{k}\right| \ll 1, K \simeq \chi\left[\cos \vartheta_{k}+(s-\right.$ 1) $\left.\sin \vartheta_{k}\left(\eta-\vartheta_{k}\right)+(s-1 / 2) \cos \vartheta_{k}\left(\eta-\vartheta_{k}\right)^{2}\right]$, Eq. (4) is Weber equation form

$$
\left\{\frac{d^{2}}{d \eta^{2}}+g(\omega)+h(\omega)\left(\eta-\vartheta_{1}\right)^{2}\right\} f=0,
$$

where $\vartheta_{1}=\vartheta_{k}-\frac{\chi(s-1) \sin \vartheta_{k}}{k^{2} s^{2} \omega+2 \chi(s-1 / 2) \cos \vartheta_{k}}, g(\omega)=$ $q^{2} k^{2}\left\{\omega^{2} \frac{\omega\left(1-i \delta_{e}\right)-\epsilon_{n}^{-1}}{\omega+\eta_{s} \epsilon_{n}^{-1}}+\omega^{2} k^{2}+2 \omega \chi \cos \vartheta_{k}-\right.$ $\left.\frac{\omega\left[\chi(s-1) \sin \vartheta_{k}\right]^{2}}{k^{2} s^{2} \omega+2 \chi(s-1 / 2) \cos \vartheta_{k}}\right\}, \quad h(\omega)=q^{2} k^{2}[2 \omega \chi(s-$ $\left.1 / 2) \cos \vartheta_{k}+k^{2} s^{2} \omega^{2}\right]$. The solutions are $f=H_{l}\left(\sqrt{-h}\left(\eta-\vartheta_{1}\right)\right) e^{-\sqrt{-h}\left(\eta-\vartheta_{1}\right)^{2} / 2}$, where $H_{l}$ is $l$-th $\left(l=l_{\eta}=0,1,2, \cdots\right)$ Hermite polynomial, and $g=(2 l+1) \sqrt{-h}$, i.e., $g^{2}+(2 l+1)^{2} h=0$, gives

$$
\begin{aligned}
& F(\omega)=q^{2} k^{2} \omega\left\{\omega\left[\omega\left(1-i \delta_{e}\right)-\epsilon_{n}^{-1}\right]\left(k^{2} s^{2} \omega+\alpha_{2}\right)+\right. \\
& \left.\left(\omega k^{2}+\alpha_{3}\right)\left(\omega+\eta_{s} \epsilon_{n}^{-1}\right)\left(k^{2} s^{2} \omega+\alpha_{2}\right)-\left(\omega+\eta_{s} \epsilon_{n}^{-1}\right) \alpha_{1}\right\}^{2} \\
& +(2 l+1)^{2}\left(k^{2} s^{2} \omega+\alpha_{2}\right)^{3}\left(\omega+\eta_{s} \epsilon_{n}^{-1}\right)^{2}=0
\end{aligned}
$$

where $\alpha_{1}=\left[\chi(s-1) \sin \vartheta_{k}\right]^{2}, \alpha_{2}=2 \chi(s-1 / 2) \cos \vartheta_{k}$, $\alpha_{3}=2 \chi \cos \vartheta_{k}$. Similar analytical solutions with $l=0$ or $\vartheta_{k}=0$ are discussed in Refs. 4, 16, 28, for different purposes of usage. Eq.(7) can be written to a seventh order polynomial (Appendix A gives its coefficients at $\vartheta_{k}=0$, which yields a fifth order polynomial) which contains seven solutions. However, only three of them can satisfy the decaying (depend on $\sqrt{-h}$ ) boundary condition, and other explosive solutions should be dropped. The above analytical solutions also tell us that the mode (e.g., ITGs) can have both even and odd parities, which is determined by quantum number $l$ (or, $l_{\eta}$ ) of the Hermite polynomial $H_{l}$. In numerical aspect, larger $N_{\eta}$ is required to make larger $l$ solutions convergent. The good news is that we are mainly interested in small $l$ solutions, which can usually be numerically handled well. The distribution of all solutions will change for larger $l$ modes but have been convergent for small $l$ modes in Fig,1a, i.e., $N_{\eta}=768$ is sufficient for that case.

Table I compares the analytical solutions of the approximated equation with the numerical solutions of the original eigen equation. We can see that with the approximation of $K$ in Eq. (5) the analytical solution can agree exactly with numerical one. However, say for $l=0$, another two explosive solutions exist in Eq. A1 but not in Eq. (5). Another difference is that the $l=1$ TEM solution exists in Eq. A1 but also can not be found (see Fig 1 in Eq. (5) (however, $l=0$ TEM can be found). This is due to the oscillation boundary condition $f \sim e^{-i \sqrt{h} \eta^{2} / 2}$, i.e., the numerical approach here can not treat $\gamma \lesssim 0$ TEM well. Comparing the solutions with and without approximation of $K$ in Table I, we can find that the analytical solutions have some deviations from the numerical ones, which come from the deviation from the approximation that mode localizes around $\eta \sim \vartheta_{k}$. The existence of an critical gradient for the jump of most unstable mode from $l=0$ to $l \neq 0$ is numerically confirmed in Ref. [5]. However, considering of the above analytical solution, which is only rough (with error $\gtrsim 20 \%$ ) agreement, it seems that an analytical expression for the critical gradient $\epsilon_{n}^{c}=\epsilon_{n}^{c}(s, k, q, \cdots)$ is challenging, let alone calculating it in more accurate kinetic model. This is one of the reason why we choose a simple model to do the analysis, which can yield better general (though not accurate) understandings. Otherwise, more powerful mathematical approaches are required. However, the major drawback of the simplified model Eq. 11) is lacking of Landau damping effect thus can not describe high $l$ solutions (which usually have larger $k_{\|}$thus will easily be damped or stabilized) correctly. A more accurate kinetic model with velocity space integral which contains Landau damping effect will be discussed in SecVI, which can give a better prediction of the critical jump gradient.

\section{B. Global analytical solution}

Eq. (4) gives local solution $\omega=\omega\left(\vartheta_{k}, x\right)$. We consider the global solution with $\partial \omega /\left.\partial x\right|_{x=0}=0$. Considering higher order ballooning representation, the second dimension equation 18 is

$$
\frac{1}{2} \frac{\partial^{2} \omega}{\partial \vartheta_{k}^{2}} \frac{d \bar{A}^{2}}{d x^{2}}+k_{\theta}^{2} s^{2}[\Omega-\omega(x)] \bar{A}(x)=0,
$$

where $\Omega$ is global eigen frequency, $\vartheta_{k}=\vartheta_{m}$ is the stationary position of local $\omega\left(\vartheta_{k}\right)$, and usually $\vartheta_{m}=0, \pi$ due to symmetry. There also exists another type of higher-order 


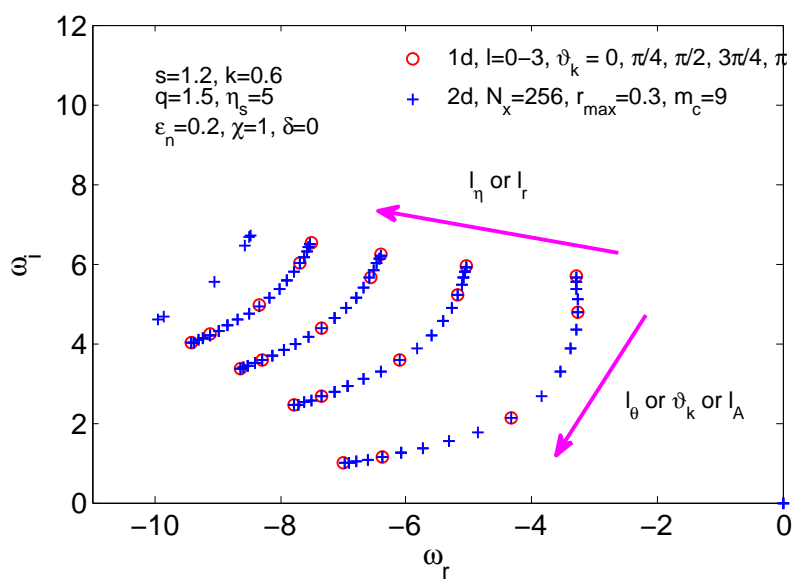

FIG. 2: Surprising relations (e.g., $l_{\eta}^{1 d} \leftrightarrow l_{r}^{2 d}$ not $l_{\theta}^{2 d}$ ! However, this can be explained by below theory) between $1 \mathrm{D}$ and $2 \mathrm{D}$ solutions, agree well for $l_{\eta}=0-3, \vartheta_{k}=0, \pi / 4, \pi / 2,3 \pi / 4, \pi$. The 2D solutions are calculated directly using Eq. 22, the 1D solutions are calculated using Eq. (4) with different $\vartheta_{k}$ for each case and then gathering them together. Note that not all solutions are shown.

theory [12, 13, 31, 32, which solves second order differential equation of $A\left(\vartheta_{k}\right)$ instead of $\bar{A}(x)$. However, the final solution will be similar, thus we will not discuss it too much. Further expanding $\omega(x)$ in Eq. (8) around a stationary point, i.e., $x=0, \omega(x)=\hat{\omega}+\omega_{x x} x^{2} / 2$, yields

$$
\frac{1}{2} \omega_{\vartheta_{k} \vartheta_{k}} \frac{d \bar{A}^{2}}{d x^{2}}+k_{\theta}^{2} s^{2}\left[(\Omega-\hat{\omega})-\omega_{x x} x^{2} / 2\right] \bar{A}(x)=0,
$$

\section{GLOBAL NUMERICAL SOLUTIONS}

In the above sections, we have shown some numerical solutions to compare with analytical solutions. In this section, we will discuss the numerical solutions in more details. To compare with previous works (e.g., Refs. [12, 13]), we will also adjust Eq.(2) slightly. We hope this section can provide an overview of the global solutions, and will focus on the steep gradient effects in next section.

\section{A. Basic features}

In numerical aspect, the discrete form of Eq. A2 contains $3 \times N_{x} \times N_{m}$ solutions. Again, 3 is due to $\omega^{3}, N_{x}$ is relevant to radial quantum number $l_{r}$, and $N_{m}=2 m_{c}+1$ is the number of poloidal $m \in\left[m_{0}-m_{c}, m_{0}+m_{c}\right]$ kept, which is relevant to poloidal quantum number $l_{\theta}$. To see these more clearly and also to compare with Ref. 13, we will use $\eta_{s}(x)=\eta_{m}-\eta_{g} x^{2}$ and $\epsilon_{n}(x)=$ const. in this where $\omega_{x x} \equiv \partial^{2} \omega / \partial x^{2}$ and $\omega_{\vartheta_{k} \vartheta_{k}} \equiv \partial^{2} \omega / \partial \vartheta_{k}^{2}$. This is again a Weber equation and has series solutions $\bar{A}(x)=$ $H_{l}(\sqrt{b} x) e^{-\sqrt{b} x^{2} / 2}, b=k_{\theta}^{2} s^{2} \omega_{x x} / \omega_{\vartheta_{k} \vartheta_{k}}$, and

$$
\Omega=\hat{\omega}+(l+1 / 2) \sqrt{\omega_{x x} \omega_{\vartheta_{k} \vartheta_{k}}} /\left(2 k_{\theta} s\right),
$$

where $l=l_{A}=0,1,2, \cdots$ and $l_{A}=0$ for lowest harmonic. The above solution will be used lately to understand the deviation of local solution to global solution and the twisting (triangle-like) mode structures in steep gradient. For more general cases, Eq.8 can be solved approximately by WKB 33] method.

As a first glance, we look at a special case, i.e., all profile parameters are constants with also $\epsilon_{n}(x)=$ const., which gives $\omega(x)=$ const. in Eq. (8). The solution can be simple $\Omega=\omega\left(\vartheta_{k}\right)$ and $\bar{A}(x)$ be arbitrary. The 1D and 2D numerical solutions of Eqs.(4) and (2) are compared in Fig,2, where we can find that the quantum number $l_{\eta}$ in $1 \mathrm{D}$ ballooning space should be $l_{r}$ (not $l_{\theta}$ !) in $2 \mathrm{D}$ real space, and the $1 \mathrm{D} \vartheta_{k}$ should be relevant to the $2 \mathrm{D}$ real space $l_{\theta}$ or ballooning approach $l_{A}$. In some sense, these are surprising. However, these can be understood from previous theory since that $\eta$ is relevant to radial $n q(r)-m$, and $\vartheta_{k}$ is relevant to $A(r)$. For the mode structures (see later), $l_{\eta}$ will be relevant to Fourier modes $u_{m}\left(r-r_{m}\right)$, and $l_{\theta}$ or $\vartheta_{k}$ will be relevant to envelop $A(r)$ and poloidal localization position $\theta$.

subsection. Eq. A2 can be used directly except change $\eta_{s}$ to $\eta_{s}(x)$.

Fig 3 shows solutions of a typical global $2 \mathrm{D}$ case. Local parameters $s=2.0, k=0.33, q=1.8, \epsilon_{n}=0.03$, $\eta_{s}=5.0, \delta_{e}=0.0, \chi=0.5$; global parameters $n=20$, $r_{s}=0.6, \eta_{g}=1.25 n$; grid parameters $N_{x}=256$. Most solutions in panel (a) and the mode structure in panel (b) will change if we use larger $m_{c}$, e.g., $m_{c}=5 \rightarrow 13$, which means that those solutions are not convergent. This is not surprising because the $2 \mathrm{D} m_{c}$ determines the number of solutions between the 1D $\vartheta_{k}=0$ to $\vartheta_{k}=\pi$. The $\theta_{p} \sim \pm \pi / 2$ solution has two peaks at both $\theta \sim \pi / 2$ and $-\pi / 2$, whereas $1 \mathrm{D}$ theory of $\vartheta_{k}=\pi / 2$ will give only one peak. Three convergent solutions are also shown, which agree well with ballooning $1 \mathrm{D}$ and $2 \mathrm{D}$ theory in previous sections for both frequency and mode structure, e.g., $\vartheta_{k}=0, \pi$ solutions localize at $\theta_{p} \sim 0, \pi$ and $l=1$ solution has odd parity of $\delta \phi_{m}(x)$ and $\vartheta_{k}=\pi$ solution has different envelop or phase for different $\delta \phi_{m}$.

Considering that panel (b) solution is not convergent, it is not known yet whether global solutions can really 
(a) $2 \mathrm{D}$ solutions

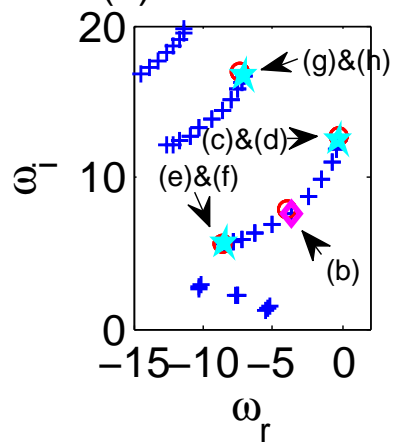

(e) $\omega=-8.41+5.59 \mathrm{i}$

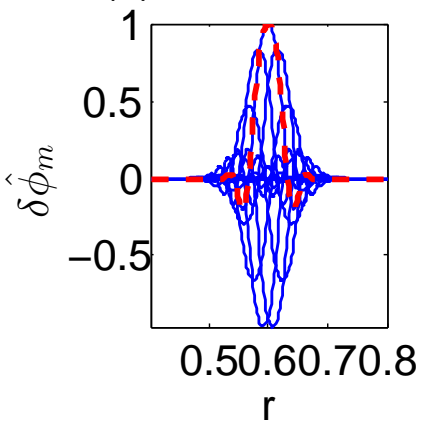

(b) $\theta_{p}=\pi / 2, l=0$

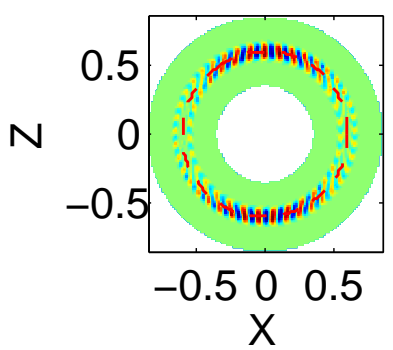

(f) $\theta_{p}=\pi, l=0$

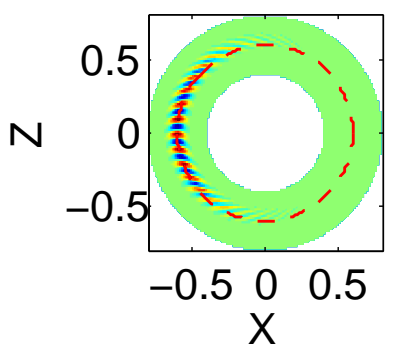

(c) $\omega=-0.178+12.4 \mathrm{i}$
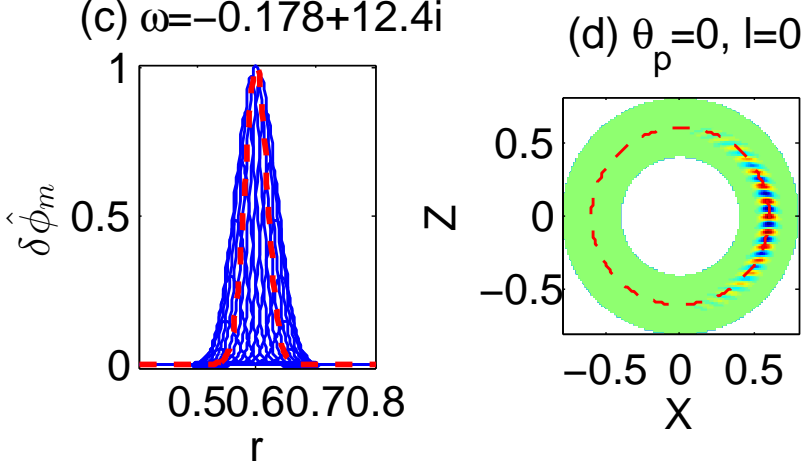

(g) $\omega=-7.04+16.7 \mathrm{i}$

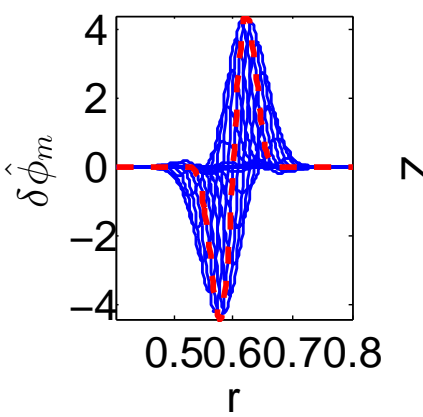

(h) $\theta_{\mathrm{p}}=0, \mathrm{l}=1$

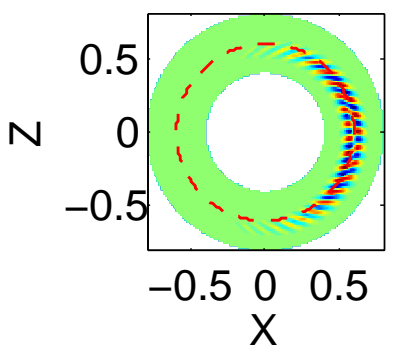

FIG. 3: Global 2D solutions, $s=2.0, k=0.33, q=1.8, \epsilon_{n}=0.03, \eta_{s}=5.0, \delta_{e}=0.0, \chi=0.5$. (a) shows series solutions, blue '+' with $m_{c}=5$. (b) $\theta_{p} \sim \pi / 2$ solution with $m_{c}=5$, corresponding $\omega$ is magenta diamond in (a). (c)-(h) for real parts of $\delta \phi_{m}$ and $\delta \phi(r, \theta)$ and cyan stars in (a), are three convergent solutions with larger $m_{c}$. Red circles in (a) are 1D solutions. Red dash lines in (c), (e), (g) are $\delta \phi_{m=m_{0}}$. Red dash lines in (b), (d), (f), (h) are $r=r_{s}$, i.e., $x=0$.

(a) $2 \mathrm{D}$ solutions, $\mathrm{m}_{\mathrm{c}}=11$
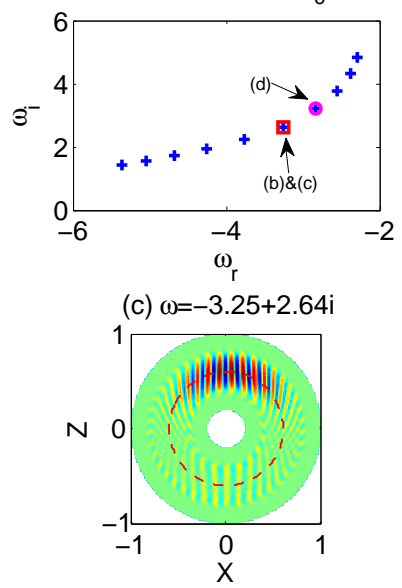

(b) $\theta_{p}=\pi / 2$


FIG. 4: Symmetry breaking from high order $O(\delta m / m)$ term leads to single peak mode at $\theta_{p}=\pi / 2$.

contains arbitrary $\vartheta_{k}$ solutions as in Fig, 2 . However, this solution still tell us that the mode structure is symmetric for $\theta=0$. This feature is similar as observed in some solutions in Ref. 5], i.e, usually mode structure will peak at both $\theta_{p} \sim \vartheta_{k}$ and $-\vartheta_{k}$.

Up to this step, we can give a short summary of ba-

sic features of unconventional modes: $\vartheta_{k}$ leads to quasicontinuous change of frequency and envelop or phase of mode structure, $l_{\eta}$ leads to discontinuous jump of frequency and high order harmonic of $\delta \phi_{m}$. The observations of global solutions in Refs. [5, 10] can be combination of both effects of $\vartheta_{k}$ and $l_{\eta}$, depending on the mode structure feature and how the frequency changes (quasi-continuous or discontinuous). Why (especially the physics behind) the most unstable solutions will change from $\vartheta_{k}=0$ and $l_{\eta}=0$ to $\vartheta_{k} \neq 0$ and $l_{\eta} \neq 0$ is out of the scope of this work, and required further study.

\section{B. Symmetry breaking}

There exists many different sources for poloidal symmetry breaking (or, up-down asymmetry) of the mode structure, i.e., away from $\theta=0$, such as rotation flow and equilibrium profile. For example, Refs. [13, 17] discuss the solutions with poloidal peaking $\theta \neq 0$ with linear profile $\eta_{s}=\eta_{m}-\eta_{g} x$ and toroidal shear flow, where poloidal tilting mode structures are shown. The tilting structure can be understood from ballooning theory as that $\vartheta_{k}=\vartheta_{m}$ where $\vartheta_{m}$ is slightly away from 0 .

In this subsection, as an example, we study the symmetry breaking from a higher order term of $\left(1 / r^{2}\right)\left(\partial^{2} / \partial \theta^{2}\right)$, i.e., treating it as $(m / r)^{2}\left(1+\delta m / m_{0}\right)^{2}$ as in Refs. [12, 31] 
instead of $(m / r)^{2}$ in SecII. The main purpose of this subsection is to show the single peak solution of $\vartheta_{k} \neq 0$.

By keeping an additional $O(\delta \mathrm{m} / \mathrm{m})$ term and dropping $i \delta_{e}$ term, the 2D Eq. (2) changes to[12]

$$
\begin{aligned}
& \left\{k^{2} s^{2} \frac{d^{2}}{d z^{2}}+\frac{1}{k^{2} q^{2} \omega^{2}}(z-m)^{2}-k^{2}-\frac{\omega-\epsilon_{n}^{-1}}{\omega+\eta_{s} \epsilon_{n}^{-1}}\right. \\
& +\underbrace{\left[\frac{k^{2}}{\omega+\eta_{s} \epsilon_{n}^{-1}}+\frac{\omega-\epsilon_{n}^{-1}}{\left(\omega+\eta_{s} \epsilon_{n}^{-1}\right)^{2}}\right] \epsilon_{n}^{-1} \eta_{s} \frac{\delta m}{m}}_{O(\delta m / m)}\} u_{m} \\
& -\chi \frac{1}{\omega}\left[\left(1+s \frac{d}{d z}\right) u_{m+1}+\left(1-s \frac{d}{d z}\right) u_{m-1}\right]=0 .
\end{aligned}
$$

The above equation can yield a similar equation as Eq. A2 and can be numerical solved in a similar manner, but with a more $\omega^{4}$ term.

Direct numerical solutions of the global Eq.(11) shown in Fig 4 confirm the ballooning theory of the second kind solution in Ref.[12], with parameters $s=1.2, k=0.6$, $q=1.5, \epsilon_{n}=0.1$ and $\eta_{s}=3.0$. However, our solution $\theta_{p}=\pi / 2$ whereas $\theta_{p}=-\pi / 2$ in Fig.5(a) of Ref. [12, which is due to a sign difference in the equation (i.e., set $\chi=-1$ in our equation can reproduce the equation in Ref.[12]). We can also see in Fig[4(d) that there also exist series other solution with peaking just slightly away from $\theta_{p}=\pi / 2$. However, in Fig, 4 , only the red square $\theta_{p}=\pi / 2$ solution is convergent and other solutions will change if we use larger $m_{c}$. It is not clear whether those unconvergent solutions can physically exist. The reason why also asymmetry mode exist in Ref. [5] [e.g., Fig.2(d),(e),(h),(i)] under strong gradient without apparent symmetry breaking source (e.g., rotation, flow) still need further study. Refs. [15, 34 have studied the finite ballooning angle $\vartheta_{k}$ effects via gyrokinetic model which are possible to provide some hints to understand the source of symmetry breaking.

To this step, the unconventional mode structure with single peak $\theta \simeq \pi / 2$ or $-\pi / 2$ in Refs. [12, 13] are not the one reported in Ref. [5]. Because that the solutions in Refs. [12, 13] is still ground state $l=0$ and the unconventional structures come only from $\vartheta_{k} \neq 0$. However, the simulation results in Ref. [5] usually show multi-peak and eigenstates jump $(l \neq 0)$, and do not have the apparent symmetric breaking sources [e.g., linear $\eta_{s}(x)=\eta_{m}-\eta_{g} x$ profile or the same high order term as in Eq.[11)] as in Refs.[12, 13].

\section{GRADIENT PROFILE EFFECT}

We study the equilibrium effect from gradient profile $L_{n}^{-1}=L_{n}^{-1}(x)$, which is more analogous to the cases of global gyrokinetic simulations in Ref. 5 . by GTC code. To model the global steep profile, we take $\epsilon_{n}^{-1}=$ $\epsilon_{n 0}^{-1} e^{-\left(r-r_{s}\right)^{2} / \Delta r^{2}}$ (Fig 5), where $\Delta r$ determines the width of the steep profile region. At strong gradient tokamak edge plasmas, $\Delta r$ can small to $0.01-0.05$.

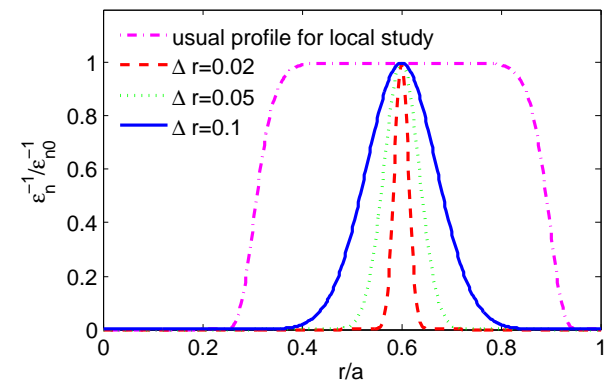

FIG. 5: The $\epsilon_{n}^{-1}=\epsilon_{n 0}^{-1} e^{-\left(r-r_{s}\right)^{2} / \Delta r^{2}}$ used for study of the global gradient profile effects. The magenta dash-dot line shows usual used profile in global code to mimic local model.

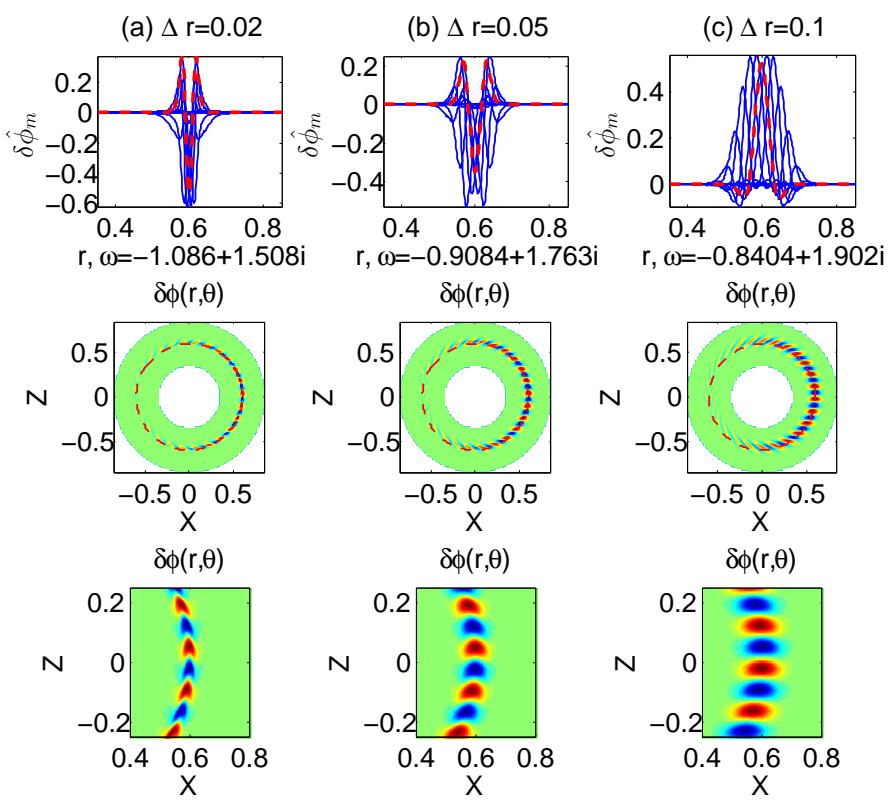

FIG. 6: Gradient profile effect $\epsilon_{n}^{-1}=\epsilon_{n 0}^{-1} e^{-\left(r-r_{s}\right)^{2} / \Delta r^{2}}$, scan $\Delta r$. Twisting (triangle-like) mode structure for smaller $\Delta r$.

The framework of analytical solutions has been discussed in Sec III. In this section, we will focus on the numerical solutions and compare them with analytical theory. Fig 6 shows typical direct numerical solutions (only fundamental $l=0$ solutions are shown) of Eq. (A2) with same local parameters $(s=1.0, k=0.3, q=2.6$, $\left.\epsilon_{n}=0.8, \eta_{s}=2.0, \delta_{e}=0.0, \chi=1.0, n=10, r_{s}=0.6\right)$ but different $\Delta r$, i.e., $0.02,0.05$ and 0.1 . We can see that smaller $\Delta r$ gives smaller growth rate $\gamma$ and also more twisting (triangle-like) of the mode structure.

These deviations of frequency and twisting of mode structure can be well understood from previous analytical theory. From local and global theory, smaller $\Delta r$ gives larger $\Omega_{x x}$ and thus larger $\Omega-\hat{\omega}$, which explains the deviation of frequency. The solutions are shown in Fig.7 and also are compared with numerical global 2D solutions. We see that analytical $\gamma$ can agree well with numerical one at $\Delta r>0.15$. For small $\Delta r$, the approximation used in the analytical theory will not be suffi- 



FIG. 7: Gradient profile effect $\epsilon_{n}^{-1}=\epsilon_{n 0}^{-1} e^{-\left(r-r_{s}\right)^{2} / \Delta r^{2}}$, scan $\Delta r$. $1 \mathrm{D}$ v.s. $2 \mathrm{D}$. Smaller $\Delta r$, smaller growth rate.


FIG. 8: Scan 1D $\omega\left(x, \vartheta_{k}\right)$, where the maximum growth rate is at $\left(x=0, \vartheta_{k}=0\right)$.

cient. Fig 7 shows that the local model can differ $50 \%$ with global model at $\Delta r=0.01$. Fig 8 shows the 1D local solutions $\omega\left(x, \vartheta_{k}\right)$, which justifies the assumption in calculating the global 2D solutions in Sec III, i.e., the maximum growth rate is stationary at $\left(x=0, \vartheta_{k}=0\right)$. The twisting mode structure is identified to come from the imaginary part of parameter $b=k_{\theta}^{2} s^{2} \omega_{x x} \omega_{\vartheta_{k} \vartheta_{k}}$ [e.g., for $\Delta r=0.04$, we have $\omega_{x x}=(-0.44-1.66 i) \times 10^{3}$, which yields $\left.b=(1.69-1.02 i) \times 10^{6}\right]$. To show the influence of $b$ to the twisting mode structure more clearly, we use $b=(0.1 \pm 1.0 i) \times 10^{6}$ to plot the global mode structures, which are shown in Fig 9 [panels (a) and (c) are difficult to distinguish, but the differences between (b) and (d) are clear]. We see that in panels (a) and (b), the twisting direction is anti-clockwise, which agrees with Fig 6 . whereas in panels (c) and (d) the sign of the imaginary part of $b$ is changed from ${ }^{\prime}-{ }^{\prime}$ to ${ }^{\prime}+{ }^{\prime}$, which leads to the twisting direction to clockwise. If $\operatorname{Im}(b)=0$, twisting structures vanish. Thus, the theory can also explain why ideal ballooning mode (IBM) in steep gradient does not have twisting radial structure, because that $\omega^{\mathrm{IBM}}=i \gamma$ and $\operatorname{Re}\left(\omega^{\mathrm{IBM}}\right)=0$. The reason why ideal Alfvén eigen modes without EP driven do not have twisting mode structure is similar, i.e., the eigen frequency $\omega$ has only real part. Considering that $k_{\theta}^{2} s^{2}$ and $\omega_{\vartheta_{k} \vartheta_{k}}$ are determined by local parameters, the global profile mainly affects $\omega_{x x}$ : smaller $\Delta r$ leads to larger $\omega_{x x}$ and then larger $\operatorname{Im}(b)$. Thus, the twisting mode structures is a global (a) $b=1 e+005-1 e+006 i$

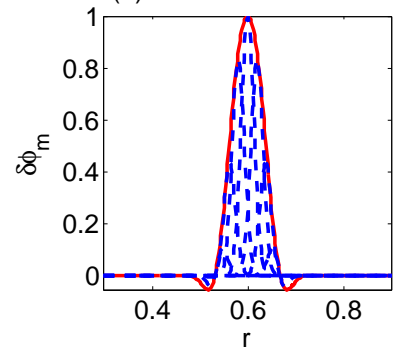

(c) $b=1 e+005+1 e+006 i$
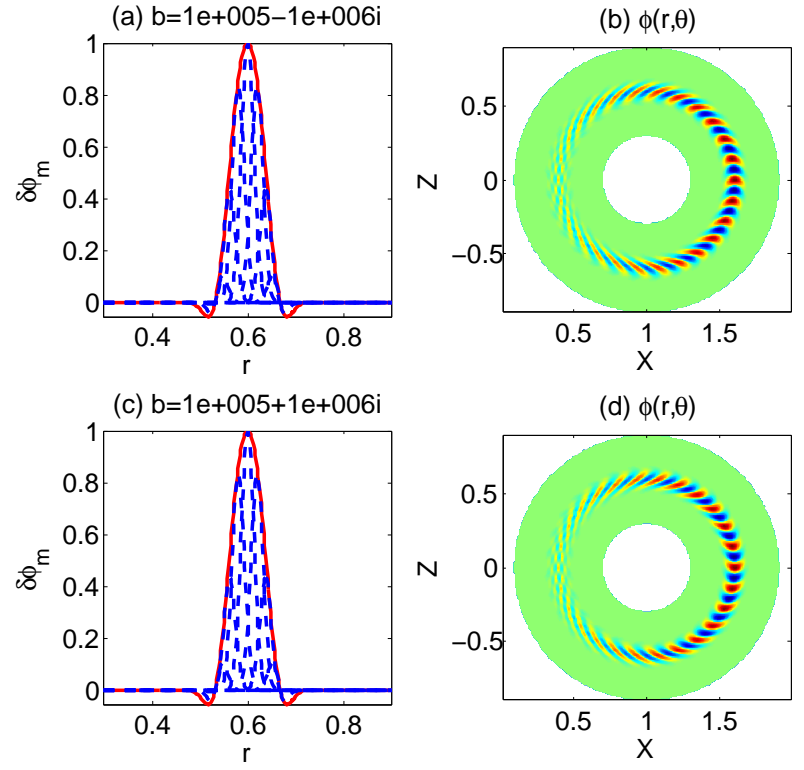

(d) $\phi(r, \theta)$

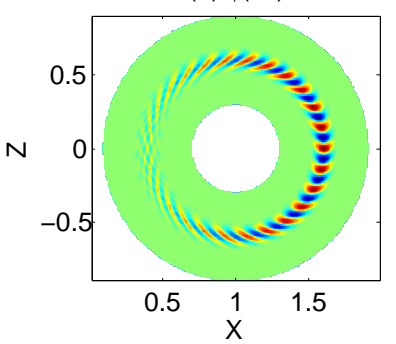

FIG. 9: The imaginary part of the parameter $b=$ $k_{\theta}^{2} s^{2} \omega_{x x} \omega_{\vartheta_{k} \vartheta_{k}}$ determines the twisting direction (clockwise or anti-clockwise).

effect which is not included in local model.

In summary, the steep gradient can lead to at least two significant global effects: deviation of the frequency and twisting of the mode structure. These global effects can not be handled well by local models and thus one should be careful in using local model to understand physics or to explain experimental observations.

\section{EIGENSTATES JUMP WITH VELOCITY SPACE INTEGRAL}

The present work is to provide more analytical insights to understand the eigenstates jump and the unconventional mode structures of drift waves. In the previous sections, we have investigated the model equation in more details than those in Ref. 5. However, a very important issue, i.e., the critical gradient $\epsilon^{c}$, is still not examined carefully. The critical gradient is confirmed to exist in Ref. [5] by the model equation. Here, we consider the case with Landau damping and with more accurate finite Larmor radius (FLR) effects, via including of the velocity space $\left(v_{\|}, v_{\perp}\right)$ integral.

We only consider ITG with adiabatic electron. The semi-local dispersion relation for ITG is 35]

$1+\frac{1}{\tau}-\left\{\frac{2}{\sqrt{\pi}} \int_{0}^{\infty} d v_{\perp} \int_{-\infty}^{\infty} d v_{\|} \frac{\left[\omega-\omega_{T}(v)\right] J_{0}^{2}\left(\sqrt{2} k_{\perp} v_{\perp}\right)}{\left[\omega-k_{\|} v_{\|}-\omega_{D}(v)\right]} v_{\perp} e^{-v^{2}}\right\}=0$,

where we have used the normalization $k_{\perp} \rightarrow k_{\perp} \rho_{i}, k_{\|} \rightarrow$ $\sqrt{2} k_{\|} L_{n}, \omega \rightarrow \omega /\left(v_{t i} / L_{n}\right), v \rightarrow v /\left(\sqrt{2} v_{t i}\right)$. Thus, after normalization, $b_{i}=k_{\perp}^{2}, \omega_{T}(v)=\omega_{* i}\left[1+\left(v_{\perp}^{2}+v_{\|}^{2}-\right.\right.$ $\left.\left.\frac{3}{2}\right) \eta_{i}\right], \omega_{D}(v)=2 \omega_{d i}\left(v_{\|}^{2}+v_{\perp}^{2} / 2\right)$, and $\omega_{* i}=k_{\perp}, \omega_{d i}=$ 

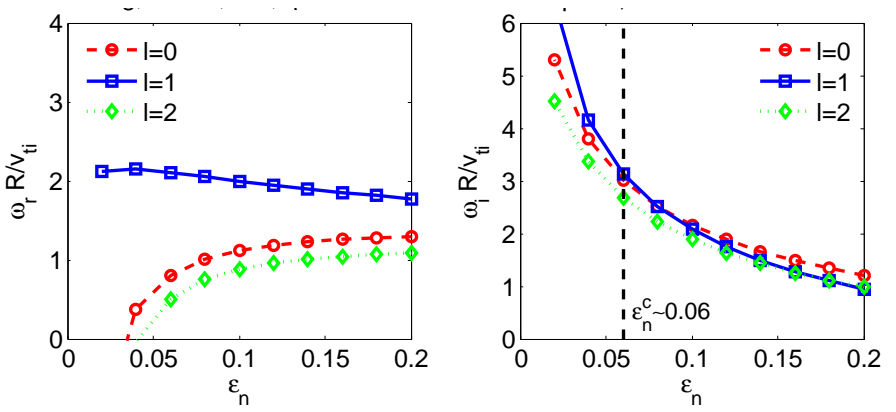

FIG. 10: Semi-local kinetic ITG dispersion relation shows the critical gradient jump of the most unstable mode from $l=0$ to $l=1$ when $\epsilon_{n}<\epsilon^{c} \sim 0.06$. Parameter $k_{\theta} \rho_{i}=0.4, \eta_{i}=3.114$, $\tau=1, s=0.78$ and $q=1.4$.

$\omega_{* i} L_{n} / R=\epsilon_{n} k_{\perp}$. In ballooning space, $k_{\perp}^{2}=k_{\theta}^{2}(1+$ $\left.s^{2} \eta^{2}\right), \partial / \partial \eta \rightarrow i q R k_{\|}$, and $\omega_{D}(v, \eta)=2 \epsilon_{n} \omega_{* i}(\cos \eta+$ $s \eta \sin \eta)\left(v_{\perp}^{2} / 2+v_{\|}^{2}\right)$.

TABLE II: Parameters for semi-local model.

\begin{tabular}{c|ccc}
\hline \hline- & $\left\langle k_{\|}^{2}\right\rangle / k_{c}^{2}$ & $\left\langle k_{\perp}^{2}\right\rangle / k_{\theta}^{2}$ & $\left\langle\omega_{D}\right\rangle /\left(2 \epsilon_{n} \omega_{* i}\right)$ \\
\hline$l=0$ & $1 / 3$ & $1+3 s^{2} / 4$ & $(1+0.75 \mathrm{~s}) / \exp (3 / 8)$ \\
$l=1$ & 1 & $1+9 s^{2} / 4$ & $(4+27 \mathrm{~s}) / 16 / \exp (3 / 8)$ \\
$l=2$ & $81 / 55$ & $1+5 s^{2} / 12$ & $(228+91 \mathrm{~s}) / 192 / \exp (3 / 8)$ \\
\hline \hline
\end{tabular}

For semi-local theory, we use trial function of mode structure $\phi(\eta)$ [i.e., $f(\eta)$ in Sec II] and calculate the local parameters by 36 .

$$
\begin{gathered}
\left\langle k_{\|}^{2}\right\rangle=-k_{c}^{2} \int \phi^{*} \frac{d^{2} \phi}{d \eta^{2}} d \eta / \int \phi^{*} \phi d \eta \\
\left\langle k_{\perp}^{2}\right\rangle=k_{\theta}^{2} \int \phi^{*}\left(1+s^{2} \eta^{2}\right) \phi d \eta / \int \phi^{*} \phi d \eta \\
\left\langle\omega_{D}\right\rangle=\int \phi^{*}\left[2 \epsilon_{n} \omega_{* i}(\cos \eta+s \eta \sin \eta)\right] \phi d \eta / \int \phi^{*} \phi d \eta,
\end{gathered}
$$

where $k_{c}=1 / q R$ and we will use $k_{\|}=\sqrt{\left\langle k_{\|}^{2}\right\rangle}$ to do the calculation. The mode structures for different eigenstates $l$ are chosen based on the analytical solution in Sec III as Hermite functions $f_{l}(\eta)=H_{l}(\sqrt{-h} \eta) e^{-\sqrt{-h} \eta^{2}}$, where we set $\sqrt{-h}=1 / 3$. The corresponding $\left\langle k_{\|}^{2}\right\rangle,\left\langle k_{\perp}^{2}\right\rangle$ and $\left\langle\omega_{D}\right\rangle$ are calculated in Table II for $l=0,1,2$. For these parameters, the above semi-local model can give similar $l=0$ ITG solutions v.s. $k_{\theta} \rho_{i}$ as those in the Fig. 1 in Ref. 8]. Actually, the above approach [e.g., solving $\phi(\eta)$ from theoretical model and use it to calculate $\left.\left\langle k_{\|}^{2}\right\rangle\right]$ is also used to study the seed parallel Reynolds stress 12 and parallel momentum transport [16].

Fig 10 shows a typical numerical solutions of Eq. (12). The velocity integral is numerical calculated by adaptive
Simpson approach. The semi-quantitative critical gradient jump of the most unstable mode from $l=0$ to $l=1$ is $\epsilon_{n}<\epsilon^{c} \sim 0.06$ and thus the critical temperature gradient parameter $R L_{T}^{-1}=\eta_{i} / \epsilon^{c} \sim 50$ (note that we have fixed $\eta_{i}$, thus we have not distinguished the separate effects of the density gradient or temperature gradient). This value is close to the simulation jump gradient in Ref. [5], i.e., $R L_{T}^{-1}=40-80$. This gradient is quite large and mainly exist at edge regions.

The above simplified calculations also show very similar jump behavior as that by a more comprehensive 1D scanning 37. of parameters for unconventional ITGs using HD7 38 code. Considering that to obtain the quantitative (especially the global) critical gradient is still challenging due to sensitive of numerical model as mentioned in Sec I those discussions are out of the scope of the present study.

\section{SUMMARY AND DISCUSSION}

In this work, we solve a global $2 \mathrm{D}$ drift wave model equation to understand the general features of drift wave in steep gradient, which is particular important to understand the edge plasmas physics (e.g, the high confinement mode). Analytical solutions in fluid limit for 1D ballooning and 2D Fourier are compared and agree closely with numerical solutions. The unconventional drift modes can be understood by two parameters: $\vartheta_{k}$ (leads to quasicontinuous change of frequency and envelop or phase of mode structure) and $l_{\eta}$ (leads to discontinuous jump of frequency and high order harmonic of $\delta \phi_{m}$ ). The steep gradient profile can largely change the local solution by causing both deviation of frequency and twisting of mode structure. The theory can also explain the twisting direction, which is determined by the imaginary part of $b=k_{\theta}^{2} s^{2} \omega_{x x} \omega_{\vartheta_{k} \vartheta_{k}}$. To give a more accurate calculation of the critical jump gradient, we also show the kinetic solutions of a semi-local model with velocity space integral, which gives critical jump temperature parameter $R L_{T}^{-1} \sim 50$ and are close to the gyrokinetic simulation value $R L_{T}^{-1}=40-80$ in Ref. [5].

The present work may be considered as a starting point to understand the drift wave in steep gradient. And future works can include: using more accurate kinetic model, calculating quasi-linear diffusion, studying the linear and nonlinear consequences. However, those works may not be straightforward. For example, the more accurate kinetic models are usually difficult to obtain complete solutions due to the lacking of powerful numerical approach or limitation of computation time. Two possible approaches have been used to solve kinetic model with complete solutions in given complex domain: Nyquist contour integral method (cf. GLOGYSTO solver in [39]) and transformation method base on Padé approximation (cf. PDRK solver in [40]). The quasi-linear study in Ref. 4 should also be extended by including global mode structure variation. Although the model used here is sim- 
ple and analytical approach here is standard, the physical understanding behind is not that trivial as first glance. We should also emphasize that the linear physics here is merely a first step to understand the future study of the more important and interesting nonlinear consequences (preliminary studies can be found at, cf. Ref. [41, 42]).

\section{Acknowledgments}

Discussions and communications with D. R. Ernst, H. T. Chen, C. J. McDevitt, D. Dickinson, T. Xie, Z. X. Lu, L. Chen, Y. Xiao and J. Q. Li are acknowledged. The work was supported by the China Postdoctoral Science Foundation No. 2016M590008 and the ITER-China Grant No. 2013GB112006.

\section{Appendix A: Polynomial form}

The polynomial form can tell us how many solutions exist in the system and also all of them can be obtained by standard numerical approach.

\section{1D analytical solution}

Polynomial form of Eq. (7) is

$$
\sum_{j} a_{j} \omega^{j}=0
$$

where, for $\vartheta_{k}=0, j=0,1, \cdots, 5, a_{0}=(1+2 l)^{2} \alpha_{2} \epsilon_{n}^{-2} \eta_{s}^{2}$, $a_{1}=\epsilon_{n}^{-1} \eta_{s}\left\{2(1+2 l)^{2} \alpha_{2}+k^{2}\left[s^{2}(1+2 l)^{2}+q^{2} \alpha_{3}^{2}\right] \epsilon_{n}^{-1} \eta_{s}\right\}$, $a_{2}=(1+2 l)^{2} \alpha_{2}+2 k^{2} \epsilon_{n}^{-1} \eta_{s}\left[s^{2}(1+2 l)^{2}+q^{2} \alpha_{3}\left(-\epsilon_{n}^{-1}+\right.\right.$ $\left.\left.\alpha_{3}+k^{2} \epsilon_{n}^{-1} \eta_{s}\right)\right], a_{3}=k^{2} s^{2}(1+2 l)^{2}+k^{2} q^{2} \epsilon_{n}^{-2}+k^{2} q^{2}\left\{\alpha_{3}^{2}+\right.$ $\left.k^{2} \epsilon_{n}^{-2} \eta_{s}\left(-2+k^{2} \eta_{s}\right)+2 \alpha_{3} \epsilon_{n}^{-1}\left[-1+\left(1+2 k^{2}-i \delta_{e}\right) \eta_{s}\right]\right\}$, $a_{4}=2 k^{2} q^{2}\left(1+k^{2}-i \delta_{e}\right)\left(-\epsilon_{n}^{-1}+\alpha_{3}+k^{2} \epsilon_{n}^{-1} \eta_{s}\right), a_{5}=$ $k^{2} q^{2}\left(1+k^{2}-i \delta_{e}\right)^{2}$. For $\vartheta_{k} \neq 0, j=0,1, \cdots, 7$, the coefficients $a_{j}$ can also be obtained straightforwardly, but are too long. Thus we do not list them here.

\section{2D equation}

Polynomial form of Eq. 2 is

$$
\begin{aligned}
& \omega^{3}\left[k^{2} s^{2} \frac{d^{2}}{d z^{2}}+i \delta_{e}-k^{2}-1\right] \phi_{m}(z)+ \\
& \omega^{2} \epsilon_{n}^{-1}\left[\eta_{s} k^{2} s^{2} \frac{d^{2}}{d z^{2}}-\eta_{s} k^{2}+1\right] \phi_{m}(z)+ \\
& \omega\left[\frac{1}{k^{2} q^{2}}(z-m)^{2}\right] \phi_{m}(z)+\epsilon_{n}^{-1}\left[\eta_{s} \frac{1}{k^{2} q^{2}}(z-m)^{2}\right] \phi_{m}(z)- \\
& \omega^{2} \chi\left[\left(1+s \frac{d}{d z}\right) \phi_{m+1}(z)+\left(1-s \frac{d}{d z}\right) \phi_{m-1}(z)\right]- \\
& \omega \eta_{s} \chi \epsilon_{n}^{-1}\left[\left(1+s \frac{d}{d z}\right) \phi_{m+1}(z)+\left(1-s \frac{d}{d z}\right) \phi_{m-1}(z)\right]=0,
\end{aligned}
$$
where $\epsilon_{n}^{-1}=\epsilon_{n}^{-1}(x)$. The above equation is solved numerically in the article with zero boundary condition.
[1] W. Horton, Rev. Mod. Phys., 71, 735 (1999).

[2] L. D. Pearlstein and H. L. Berk, Phys. Rev. Lett., 23, 220 (1969).

[3] L. Chen and C. Z. Cheng, Phys. Fluids, 23, 2242 (1980).

[4] W. Horton, D. Choi and W. M. Tang, Phys. Fluids, 24, 1077 (1981).

[5] H. S. Xie and Y. Xiao, Phys. Plasmas, 22, 090703 (2015).

[6] D.R. Ernst, K. Zeller, N. Basse, L. Lin, M. Porkolab, W. Dorland, and A. Long, Bull. Am. Phys. Soc., 50235 (2005). http://www-internal.psfc.mit.edu/research/alcator/ pubs/APS/APS2005/ernst.pdf

[7] E. Wang, X. Xu, J. Candy, R. Groebner, P. Snyder, Y. Chen, S. Parker, W. Wan, G. Lu, and J. Dong, Nucl. Fusion, 52, 103015 (2012).

[8] G. Rewoldt, Z. Lin and Y. Idomura, Computer Physics Communications, 177, 775 (2007).

[9] J. Candy, Phys. Plasmas, 12, 072307 (2005).

[10] D. P. Fulton, Z. Lin, I. Holod and Y. Xiao, Phys. Plasmas, 21, 042110 (2014).

[11] X. Liao, Z. Lin, I. Holod, Y. Xiao, B. Li, and P. B. Snyder, "Microturbulence in DIII-D tokamak pedestal. III. Effects of collisions", Phys. Plasmas, in press, (2016).

[12] T. Xie, Y. Z. Zhang, S. M. Mahajan and A. K. Wang, Phys. Plasmas, 19, 072105 (2012).

[13] D. Dickinson, C. M. Roach, J. M. Skipp and H. R. Wil- son, Phys. Plasmas, 21, 010702 (2014).

[14] J. W. Connor and J. B. Taylor, Phys. Fluids 30, 3180 (1987).

[15] R. Singh, S. Brunner, R. Ganesh and F. Jenko, Phys. Plasmas, 21, 032115 (2014).

[16] Z. X. Lu, Phys. Plasmas, 22, 052118 (2015).

[17] P. A. Abdoul, D. Dickinson, C. M. Roach and H. R. Wilson, Plasma Phys. Control. Fusion, 57, 065004 (2015).

[18] R. Hastie, K. Hesketh and J. Taylor, Nucl. Fusion, 19, 1223 (1979).

[19] J. B. Taylor, J. Connor and H. R. Wilson, Plasma Phys. Control Fusion, 35, 1063 (1993).

[20] W. Deng, Z. Lin, I. Holod, X. Wang, Y. Xiao and W. Zhang, Phys. Plasmas, 17, 112504 (2010).

[21] X. Wang, F. Zonca, and L. Chen, Plasma Phys. Controlled Fusion 52, 115005 (2010).

[22] H. Zhang, Z. Lin, I. Holod, X. Wang, Y. Xiao, and W. Zhang, Phys. Plasmas 17, 112505 (2010).

[23] E. M. Bass and R. E. Waltz, Phys. Plasmas 20, 012508 (2013).

[24] R. Ma, F. Zonca and L. Chen, Physics of Plasmas, 22, 092501 (2015).

[25] A. Bottino, A. G. Peeters, O. Sauter, J. Vaclavik, L. Villard, and ASDEX Upgrade Team, Phys. Plasmas 11, 198 (2004).

[26] J. W. Connor, R. J. Hastie and J. B. Taylor, Phys. Rev. 
Lett., , 40, 396 (1978).

[27] J. W. Connor, R. J. Hastie and J. B. Taylor, Proc. R. Soc. London Ser.A, 365, 1 (1979).

[28] F. Romanelli and F. Zonca, Phys. Fluids B 5, 4081 (1993).

[29] J. Q. Dong, S. M. Mahajan and W. Horton, Phys. Plasmas, 4, 755 (1997).

[30] D. R. Ernst, P. T. Bonoli, P. J. Catto, W. Dorland, C. L. Fiore, R. S. Granetz, M. Greenwald, A. E. Hubbard, M. Porkolab, M. H. Redi, J. E. Rice, K. Zhurovich and Alcator C-Mod Group, Phys. Plasmas, 11, 2637 (2004).

[31] Y. Z. Zhang and S. M. Mahajan, Phys. Lett. A 157, 133 (1991).

[32] T. Xie, H. Qin, Y. Z. Zhang and S. M. Mahajan, Phys. Plasmas, 23, 042514 (2016).

[33] J. Heading, Q. J. Mech. Appl. Math. 15, 215 (1962).

[34] P. Migliano, Y. Camenen, F. J. Casson, W. A. Hornsby, and A. G. Peeters, Phys. Plasmas 20, 022101 (2013).

[35] J. Y. Kim, Y. Kishimoto, W. Horton and T. Tajima, Phys. Plasmas, 1, 927 (1994).
[36] A. Hirose, L. Zhang and M. Elia, Phys. Plasmas, 2, 859 (1995).

[37] M. K. Han, Z. X. Wang, J. Q. Dong and H. R. Du, Ion temperature gradient modes of unconventional ballooning structures in pedestal region of tokamaks, to be submitted (2016).

[38] J. Q. Dong, L. Chen, F. Zonca and G. D. Jian, Phys. Plasmas, 11, 997 (2004).

[39] S. Brunner, M. Fivaz, T. M. Tran and J. Vaclavik, Phys. Plasmas, 5, 3929 (1998).

[40] H. S. Xie and Y. Xiao, Plasma Science and Technology, 18, 97 (2016).

[41] H. S. Xie, Numerical Simulations of Micro-turbulence in Tokamak Edge, PhD thesis, Zhejiang University, (2015). http://hsxie.me/files/thesis/

[42] M. J. Pueschel, D. R. Hatch, P. W. Terry and J.W. Connor, Edge Turbulence: Mode Parity and Consequences for Transport, U.S. Transport Task Force Workshop, Salem, May, 2015. 